\title{
Conclusion:
}

During this symposium the complexities of the disease are addressed including the toll PDP can have on the person living with $\mathrm{PD}$, their care partner(s) and the interdisciplinary health care team. It is imperative clinicians can simultaneously manage the overlapping motor symptoms and PDP to prevent physical and mental disabilities and improve quality of life. After attending this session, clinicians will be able to provide quality care to individuals living with PDP and their care partners.

\section{1 - Symposium social health: a pathway to inclusion and cognitive health}

\author{
Social health, social inclusion and its associations with cognitive functioning
}

\section{Myrra Vernooij-Dassen, Eline Verspoor, Claudia Hubers, Marta Lenart, Henrik Wiegelman, Marieke Perry}

Background: Inclusion is taken as a natural situation, until feelings of exclusion are perceived. Social relations are for human beings like water to plants. Social health has been defined in 1946 by the WHO as the social domain of health. It is an umbrella concept that covers how the individual relates to his or her social environment and vice versa. Social inclusion is a key marker or characteristic of social health, represented by specific markers such as participation in leisure activities.

Objective: We aim to study theoretical mechanisms and social health markers relevant to inclusion and cognitive functioning.

Methods: identification of mechanistic pathways and systematic review on the relationship between combinations of social health markers and cognitive functioning and dementia in healthy older adults.

Results: We combined neurobiological and social pathways to guide our study. The search for social health markers yielded 4332 potentially relevant citations. Eleven articles were eligible for inclusion. Combining social health marker reflecting social exclusion (e.g. social isolation, financial deprivation, living alone and lacking basic social rights) revealed a significant risk factor for both the development of dementia and reduced cognitive functioning. A combination of a high educational level, high occupational complexity and participating in leisure activities was protective for good cognitive functioning and dementia.

Conclusion: Several social health markers are a pathway to social inclusion and to cognitive functioning, with markers reflecting exclusion being a risk factor, while those reflection inclusion are associated with protective effects. These findings open doors for interventions using the potential of social health in prevention of cognitive decline and dementia.

Social Health And Reserve in the Dementia patient journey (SHARED): Females, older adults, and people living with dementia are vulnerable to social isolation

Suraj Samtani ${ }^{1}$, Ashley Stevens ${ }^{1}$, Darren M. Lipnicki ${ }^{1}$, Erico Castro Costa ${ }^{2}$, Maëlenn Guerchet ${ }^{3}$, PierreMarie Preux ${ }^{3}$, Ingmar Skoog ${ }^{4}$, Nikolaos Scarmeas ${ }^{5,6}$, Ki-Woong Kim ${ }^{7}$, Mary Ganguli ${ }^{8}$, Michael Crowe ${ }^{9}$, Tze Pin $\mathrm{Ng}^{10}$, Katya Numbers ${ }^{1}$, Perminder S. Sachdev ${ }^{1}$, Henry Brodaty ${ }^{1,11}$ and the SHARED consortium for the Cohort Studies of Memory in an International Consortium (COSMIC)

1. Centre for Healthy Brain Ageing (CHeBA), School of Psychiatry, UNSW Medicine and Health, UNSW Sydney, Australia

2. Instituto René Rachou, Fundação Oswaldo Cruz, Belo Horizonte, MG, Brasil 
3. Tropical Neuroepidemiology, Université de Limoges, France

4. Neuropsychiatric Epidemiology Unit, Department of Psychiatry and Neurochemistry, Institute of Neuroscience and Physiology, Sahlgrenska Academy, Centre for Ageing and Health (AgeCap) at the University of Gothenburg, Sweden

5. Department of Neurology, Columbia University, New York, NY, USA

6. $1^{\text {st }}$ Department of Neurology, Aiginition Hospital, National and Kapodistrian University of Athens, Greece

7. Department of Neuropsychiatry, Seoul National University Bundang Hospital, 300 Gumiro, Bundanggu, Seongnamsi, Gyeonggido, 463-707, South Korea

8. Department of Psychiatry, Epidemiology and Neurology, University of Pittsburgh, USA

9. Department of Psychology, University of Alabama at Birmingham, AL, USA

10. National University of Singapore, Yong Loo Lin School of Medicine, Department of Psychological Medicine, Level 9, NUHS Tower Block, 1E Kent Ridge Road, Singapore 119228

11. Dementia Centre for Research Collaboration (DCRC), School of Psychiatry, Faculty of Medicine and Health, UNSW Sydney, Australia

Background: The SHARED project is an international consortium exploring the link between social factors and the onset and course of dementia across the lifespan. We investigated differences in social health variables across sex, age (40-59, 60-79, 80+ years old), and cognitive status (23768 cognitively healthy, $6944 \mathrm{MCl}$, and 1318 people living with dementia).

Method: Data was harmonised across 12 international longitudinal studies of ageing (the English Longitudinal Study of Ageing and 11 COSMIC consortium studies). The effect of age, sex, cognitive status, and their combinations at baseline on social health variables such as relationship status, living situation, frequency of interactions, and loneliness were investigated.

Result: Our results indicated that age, sex, and cognition have significant but varying effects on each of the social health variables. Examining combinations of variables revealed that females aged $80+$ were the most likely to be widowed $(O R=4.85)$, living alone $(O R=5.44)$ and to feel lonely $(O R=2.13)$. Males $(O R=1.35)$, and people living with $\mathrm{MCI}(\mathrm{OR}=2.48)$, or dementia $(\mathrm{OR}=6.63$ ) were more likely to report never interacting with friends/family.

Conclusion: Harmonised data from 12 longitudinal studies of ageing across 13 countries revealed that adults over the age of 60 (especially females) were more likely to live alone, and experience widowhood and loneliness. Similarly, people living with dementia and males were less likely to interact with friends/family. Public health policy for older adults must target these vulnerable groups to build pathways towards social inclusion.

\section{Social health during the COVID-19 pandemic: The Rotterdam Study}

Author List: Isabelle F. van der Velpen ${ }^{1,2}$, Rowina F. Hussainali ${ }^{1,3,4}$, René J.F. Melis ${ }^{5,6}$, Marieke Perry ${ }^{5,7}$, Myrra J.F. Vernooij-Dassen ${ }^{8}$, M. Arfan Ikram ${ }^{1}$, Annemarie I. Luik $^{1,9}$, Meike W. Vernooij ${ }^{1,2}$

1. Department of Epidemiology, Erasmus MC, Rotterdam, The Netherlands

2. Department of Radiology and Nuclear Medicine, Erasmus MC, Rotterdam, The Netherlands

3. Generation R Study Group, Erasmus MC, Rotterdam, The Netherlands

4. Department of Obstetrics and Gynecology, Erasmus MC, Rotterdam, The Netherlands

5. Department of Geriatric Medicine, Radboudumc Alzheimer Center, Radboud University Medical Center, Nijmegen, The Netherlands

6. Radboud Institute for Health Sciences, Radboud university medical center, Nijmegen, The Netherlands 


\section{Department of Primary and Community Care, Radboud university medical center, Nijmegen, The Netherlands \\ 8. Department of IQ Healthcare, Radboud university medical center, Nijmegen, The Netherlands \\ 9. Department of Child and Adolescent Psychiatry/Psychology, Erasmus MC, The Netherlands}

Background: The COVID-19 pandemic changed social life significantly. Sociodemographic factors and social health prior to the pandemic may determine how the changes in social life were perceived. Importantly, brain structure has increasingly been linked to social health and may play a role in how older adults perceived the change in social health during the pandemic.

Research Objective: To study the effect of brain structure prior to the COVID-19 pandemic on social health during the pandemic in community-dwelling older adults.

Method: Social health markers (loneliness, perceived social support) and sociodemographic data were assessed in the Rotterdam Study from 2012 to 2019. Participants underwent magnetic resonance imaging of the brain between 2005 and 2017. Global brain volumes, cerebral small vessel disease markers and white matter microstructural integrity were used as markers of structural brain health. On April $20^{\text {th }} 2020$, a COVID-19 questionnaire was sent out to all living Rotterdam Study participants $(N=8,732)$. The questionnaire included a shortened version of the Center for Epidemiological Studies Depression Scale (CES-D), the 3-item UCLA Loneliness scale and items on social connectedness. Two follow-up questionnaires were sent out in May 2020. Linear mixed models will be used to study the associations between brain structure and social health markers during the COVID-19 pandemic.

Preliminary results of the ongoing study: Social health data during the COVID-19 pandemic were complete for 5,020 participants (mean age: 68.7 (SD 11.3); 56.8\% women). Mean UCLA loneliness score was 4.6 (SD 1.6). Prevalence of loneliness measured on CES-D was $27.9 \%$ in April 2020, compared to $13.2 \%$ during the last Rotterdam Study follow-up round. Loneliness prevalence was higher among women (35.0\%), single participants (49.1\%) and those living alone (55.1\%). Participants felt connected to their neighbors, family and friends $(89.7 \%)$ and to a lesser extent to all Dutch people $(65.4 \%)$.

Conclusion: Community-dwelling older adults in Rotterdam experienced more loneliness in the first weeks of the COVID-19 pandemic in 2020, but also reported feeling socially connected to their loved ones and community. Using longitudinal analyses, we will next explore how brain structure affected social health during the first wave of the pandemic.

\section{Social inclusion, health services and people with young-onset dementia, a path still to develop}

Rabih Chattat, Ilaria Chirico, Alessandro Pappadà, Giovanni Ottoboni

Department of Psychology, University of Bologna, Italy

Background: The capacity to record and act proactively towards the needs of people with dementia is not equally distributed across Europe. In particular, when young people receive the diagnosis of dementia, social participation and social roles require special attention. We explore health services' recognition of the need for social participation and subsequent actions.

Methods: People with dementia, formal and informal caregivers (health service workers) from Italy, were interviewed about their experiences with people with young-onset dementia. The transcripts were re-analysed focussing on social inclusion and using qualitative content analysis. 
Results: Participants were twenty-two people living with young-onset dementia, fifty-one informal carers and thirty-one formal caregivers. Four themes emerged: (1) impact; (2) coping; (3) service internal functioning; (4) service networking.

The affected people's young age was crucial as it increased the discrepancy between their sentiments of usefulness and inclusion in the society and the attitude of health services and caregivers. Maladaptive coping strategies were put in place as reactions to the diagnosis and in response to the absence of either structures or plans capable of re-uptaking and empowering these people.

Conclusions: Young-onset dementia represents a dramatic turn-of-the-screw in all the people impacted by this diagnosis. One of the needs people living with young-onset diagnosis report relates to social inclusion and maintaining a social role. Considering social needs and enhancing interpersonal interactions and mutual recognition can positively impact individuals' quality of life as it can buffer their limitations and enhance their capabilities.

\section{2 - The global impact of the COVID-19 pandemic on those living with and caring for someone with dementia}

Clarissa Giebel, Marta Lenart, Ilaria Chirico, Katarzyna M. Lion

We are presenting four abstracts here for four inter-related talks into the global impact of the pandemic on dementia. Specifically, we will be presenting four talks from our international consortium (UK, India, Italy, Poland, Australia) highlighting how dementia has increased as a global public health concern during the pandemic with similar and different impacts across countries; the mental health impact on people with dementia and carers; the difficulties in using technology for social contact in dementia; as well as the impact on carers with relatives residing in institutional long-term care settings. Public involvement has been a key element of this study. The symposium will be hosted by Dr Clarissa Giebel, the PI of this international 5-country study, and talks will be presented by Dr Giebel, Marta Lenart, Dr Ilaria Chirico, and Dr Katarzyna Lion.

\section{Talk 1 Abstract}

Amplifying dementia as a global public health problem: A cross-country comparison of the impact of COVID-19 pandemic

\section{Clarissa Giebel ${ }^{1,2^{*}}$, Katarzyna Lion ${ }^{3}$, Maria Mackowiak ${ }^{4}$, Rabih Chattat ${ }^{5}$, PN Suresh Kumar ${ }^{6}$, Monica} Cations $^{7}$, Mark Gabbay ${ }^{1,2}$, Wendy Moyle ${ }^{3}$, Giovanni Ottoboni ${ }^{5}$, Joanna Rymaszewska ${ }^{4}$, Adrianna Senczyszyn ${ }^{4}$, Dorota Szczesniak ${ }^{4}$, Hilary Tetlow ${ }^{2}$, Elzbieta Trypka ${ }^{4}$, Marco Valente ${ }^{5}$, Ilaria Chirico ${ }^{5}$

1 Department of Primary Care \& Mental Health, University of Liverpool, UK 2 NIHR ARC NWC, Liverpool, UK

3 Menzies Health Institute Queensland, Griffith University, Australia

4 Department of Psychiatry, Wroclaw Medical University, Poland 5 Department of Psychology, University of Bologna, Italy 6 IQRAA International Hospital and Research Center, Kerala, India 7 College of Education, Psychology and Social Work, Flinders University, Australia

Background: Emerging evidence describes impacts of the COVID-19 pandemic upon people living with dementia and their informal carers, however without evidence-based global comparisons to date. The aim of this international study was to explore and compare the impact of COVID-19 and associated public health restrictions on the lives of people living with dementia and informal carers and access to dementia care across five countries. 